\title{
Perceptual processing at adjacent locations on a single finger: Masking and response competition
}

\author{
DAVID T. HORNER \\ University of Wisconsin, Oshkosh, Wisconsin
}

\begin{abstract}
When target patterns and nontarget patterns are presented either to the same or to adjacent locations on the distal pad of the index finger, the amount of interference in identifying targets depends on both the shape and the location of the nontarget (Horner, 1997). In the present study, the question of whether such interference is caused by masking (the masker in some way distorts the initial representation of the target) or by response competition (the observer mistakenly responds with the masker, rather than with the target) was investigated. A 4-to-2 paradigm was used (Craig, 1995), in which four stimuli were mapped to only two responses. Targets and nontargets were randomly selected from the set of four stimuli and presented to the same or adjacent locations on the same fingerpad. Both the distal pad and the medial pad of the index finger were tested, because innervation density varies proximodistally on the distal pad, but not on the medial pad. The results indicated that response competition was an important factor limiting perception. Furthermore, perception was affected by varying location on the distal pad, but not on the medial pad. Finally, varying location on the distal pad affected perception only when responses were based on pattern shape, not when responses were based on direction of motion. The results are discussed in terms of differences in innervation density between adjacent locations and possible resultant differences in the spatial filtering properties of the skin.
\end{abstract}

An observer who is inspecting an object tactually may gain valuable information by identifying individual features on the object's surface. Such features contact the fingerpad sequentially as the fingers are moved along the object's surface, and identifying an individual feature may require separating it temporally from other features. This type of tactual exploration resembles a temporal masking task, in which an observer identifies a target pattern in the presence of a masker that either precedes or follows it. Furthermore, in tactual exploration, separate surface features may contact different locations on a single fingerpad, and identifying a feature at one location may be affected by features at other locations. Similarly, in a temporal masking task, varying the location of the masker on the fingerpad may affect the amount of interference in identifying the target pattern (Horner, 1997). This result, along with results from other pattern identification tasks involving adjacent locations on the fingerpad (Hill, 1974; Nolan \& Kederis, 1969; Weisenberger, 1981), raises the question of whether information about the shape of a tactile pattern is independent of its location.

An earlier version of this material was presented at the American Psychological Society meeting in June 1996. San Francisco. This research was supported by the University of Wisconsin Oshkosh and by Grant DC-00095 from the National Institutes of Health. The author thanks James Craig for his comments on earlier versions of this manuscript. Correspondence concerning this article should be addressed to D. T. Horner, Psychology Department, University of Wisconsin Oshkosh, Oshkosh, WI 54901-8601 (e-mail: horner(u)uwosh.edu).
To answer this question, Horner (1997) presented a target and a masker either to the same location (SLoc) or to different locations (DLoc) on the distal pad of the left index finger. Furthermore, the masker was either the same shape as the target or a different shape. The amount of interference in identifying a target depended on both the shape and the location of the masker. Specifically, when patterns were at SLoc, target identification was disrupted greatly by a different shape masker, but only slightly by an identical masker. When patterns were at DLoc, interference by a different shape masker was reduced, but interference by an identical masker increased. Because the study was concerned mainly with whether perception changed as a function of location, the cause of the interference effects was left unresolved.

The two most likely explanations for these interference effects are masking and response competition. Masking refers to interference in the identification of a target that occurs at a relatively early stage in processing, during which the masker in some way distorts the representation of the target (Craig, 1989, 1995; Evans, 1987; Evans \& Craig, 1986). Response competition refers to interference that occurs at a later stage in processing in which representations of both the target and the masker are available and the observer mistakenly responds with the masker (Craig, 1995; Evans \& Craig, 1992; Evans, Craig, \& Rinker, 1992). Both masking and response competition may occur when patterns are presented to SLoc (Craig, 1995). When patterns are presented to locations on separate fingers, response competition is a major factor limiting performance (Craig \& Evans, 1995; Evans \& Craig, 1991, 1992; Evans et al., 1992; Rinker \& Craig, 1994). No 
study has yet examined the cause of interference effects when patterns are presented to adjacent locations on a single finger.

When patterns are presented to a single finger, as in the Horner (1997) study, one might predict that both masking and response competition would be less likely to limit performance in identifying targets when maskers are at DLoc than when they are at SLoc. It is particularly difficult to imagine how either masking or response competition could account for increased interference by an identical masker presented to DLoc. Masking would be unlikely because an identical masker has the same features as the target, and response competition would be unlikely because the masker and the target have the same response. The solution to the problem may reside in the nature of the two locations tested in the Horner (1997) study, which were adjacent locations on the distal pad of the index finger. Physiological studies suggest that the density of innervation of first order afferents varies substantially in the proximodistal direction along the distal pad of the finger (Johansson \& Vallbo, 1979; Vallbo \& Johansson, 1984) and that spatial acuity also varies in the proximodistal direction (Craig, 2000). If differences in innervation density cause an identical masker at DLoc to feel different than the target (Horner, 1995), an identical masker may interfere with target processing in a manner similar to that of a different shape masker. The question then is, how does a different shape masker at DLoc interfere with target identification? Horner's (1997) results suggested that interference effects may have been due to a combination of response competition and masking.

The primary goal of the present study was to determine the relative contributions of masking and response competition when the locations of targets and nontargets were varied on a single fingerpad. A procedure called the 4-to-2 paradigm was used to separate the effects of masking and response competition (Craig, 1995; Evans \& Craig, 1991, 1992; Evans et al., 1992). As is shown in Figure 1, there were four stimuli (A, B, C, and D) but only two responses ( 1 and 2 ). Observers pressed key number $I$ in response to $\mathrm{A}$ or $\mathrm{B}$ and key number 2 in response to $\mathrm{C}$ or D. On each trial, two patterns chosen randomly from the four were presented sequentially, and the observers responded to one (the target) and attempted to ignore the other (the nontarget). There were three possible types of trials, based on the pairing of the target and the nontarget. On stimulus compatible (SC) trials, the target and the nontarget were identical shapes and had the same response (e.g., pattern A presented twice). On response compatible (RC) trials, the target and the nontarget were different shapes but had the same response (e.g., patterns A and $B$ ). On response incompatible (RI) trials, the target and the nontarget differed in shape and had different responses (e.g., patterns $\mathrm{A}$ and $\mathrm{C}$ ). If response competition occurs, performance should be reduced on RI trials, as compared with SC or RC trials, because the target and the nontarget have different responses only on RI trials. If masking occurs, performance should be reduced on RC trials, as compared with SC trials, because these two types of trials differ with respect to the shapes of the target and the nontarget, but not with respect to their responses. The amount of masking that occurs may be underestimated if observers are responding with the nontarget on RC trials, since responding with the nontarget would lead to a correct response (Craig, 2000).

A second goal of the present study was to examine the extent to which observers can attend selectively to adjacent locations on a single fingerpad. Attention is sometimes described as a spotlight highlighting a region of space that enjoys special processing capabilities (Driver
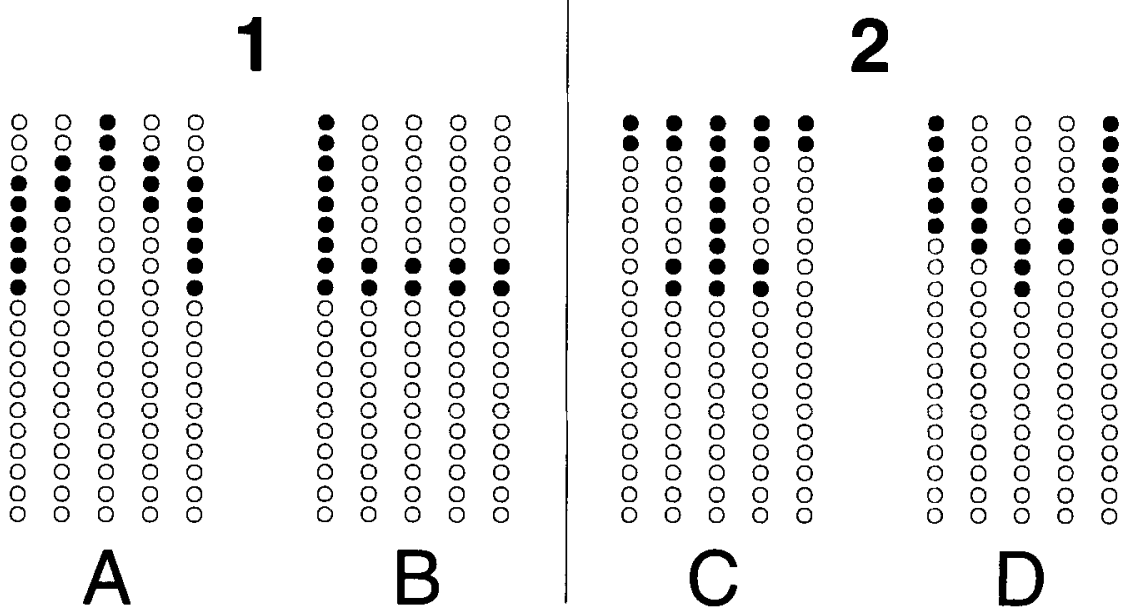

Figure 1. Representations of the patterns used as targets and nontargets in Experiment 1 as they would appear at the top of the distal pad of the finger (L1). Filled circles indicate active pins in the array, and unfilled circles indicate inactive pins. 
\& Baylis, 1991; Eriksen \& Hoffman, 1973). Stimuli within the spotlight are processed more quickly and accurately than those outside the spotlight, and the spotlight may vary in size, depending on task requirements. To the extent that attention can be restricted to the target location, observers should be less likely to respond with a nontarget presented to an adjacent location. The results of Horner's (1997) study suggested that observers were at least partly able to restrict their attention to one of two adjacent locations on the same fingerpad. The question in the present study is whether response competition is reduced when nontargets are presented to an adjacent location, rather than to the target location.

Aside from the aforementioned theoretical issues, a practical reason for varying the locations of targets and nontargets on the same finger is the improvement of tactile communication systems that rely on the ability to process successive spatial patterns, such as Braille (Nolan \& Kederis, 1969) and the Optacon (Bliss, Katcher, Rogers, \& Shepard, 1970). Braille and Optacon patterns are presented to the distal pad of the finger, and the location where patterns are felt may affect reading performance. Performance may also be affected by temporal masking (Craig, 1976, 1982, 1983; Craig \& Evans, 1987; Evans, 1987; Evans \& Craig, 1986) and by spatial interactions between successive patterns (Craig, 1980, 1989; Evans, 1987; Evans \& Craig, 1986; Loomis \& Apkarian-Stielau, 1976; Weisenberger \& Craig, 1982). Temporal masking and spatial interactions between patterns may be reduced as the physical distance between patterns on the skin increases, resulting in improved legibility.

Experiment 1 used the 4-to-2 paradigm with stationary spatial patterns similar to those in Horner's (1997) study to compare the effects of masking and response competition when varying location on the distal pad of the index finger. Stationary spatial patterns have been used previously in studies of temporal masking (Craig, 1976, 1982, 1985; Evans, 1987; Evans \& Craig, 1986). The distal pad of the finger was selected not only because it was used in the Horner (1997) study, but also because of its role in both tactual exploration and the processing of successive spatial patterns in tactile communication systems, such as Braille and the Optacon (Bliss et al., 1970). In Experiment 2, the effects of masking and response competition were compared by using moving barshaped patterns, with which responses were based on perceiving direction of motion, not shape. Experiment 3 tested stationary patterns on the medial pad of the index finger to compare the effects of masking and response competition, using locations that do not vary substantially in density of innervation (Johansson \& Vallbo, 1979; Vallbo \& Johansson, 1984).

\section{EXPERIMENT 1}

The primary question in Experiment 1 was whether varying the location of the nontarget would affect performance levels on SC, RC, and RI trials. Craig (1995) used the 4-to- 2 paradigm and presented stationary spatial patterns to SLoc on the distal pad of the left index finger. He found different results, depending on the nature of the pattern set he used. When he used simple barshaped patterns containing no line segments in common, there was little difference in performance between SC and $R C$ trials, but there was a difference between $R C$ and $\mathrm{RI}$ trials, suggesting that little masking occurred but that response competition interfered with target identification. However, when he used a set of more complex patterns, with which each feature of the pattern had to be perceived for the pattern to be identified correctly, he found that masking occurred.

The patterns used in Experiment 1 (Figure 1) were more complex than Craig's (1995) simple bar-shaped patterns but did not share the same qualities as his more complex pattern set. Therefore, it was difficult to predict whether masking would limit performance when the patterns in Experiment 1 were presented to SLoc. However, on the basis of Craig's results, it was assumed that response competition would interfere with target identification when patterns were at SLoc. The main question was whether any effects that were due to masking or response competition would change when patterns were presented to DLoc. If changing location on the distal pad of the finger affects the perceived shape of patterns, performance on SC and RC trials may be lower when patterns are at DLoc, because the nontarget may evoke a response different from that of the target. On the other hand, performance on RI trials may improve when patterns are at DLoc, because the nontarget may be less likely to evoke a competing response if it is perceived as a different shape.

\section{Method}

Participants. Five female and 2 male students served as trained observers: all had received practice in tasks similar to the present one. The observers either were paid or received course credit for participation.

Apparatus. An IBM PS/ValuePoint 466DX2 computer controlled the duration of vibration of the individual pins in the vibratory array of the Optacon II. a reading aid for the blind (Bliss et al., 1970). The vibratory array measures $9.6 \mathrm{~mm}$ wide by $22.8 \mathrm{~mm}$ high and consists of 100 blunt pins arranged in 20 rows $(1.2 \mathrm{~mm}$ separating the rows) and five columns ( $2.4 \mathrm{~mm}$ separating the columns). Each of the 0.25 - $\mathrm{mm}$-diameter pins vibrated at 230 pulses per second and at a comfortable intensity well above threshold. The vibratory array contacted the distal pad of the left index finger.

Stimuli. The targets and nontargets were randomly selected, with replacement from the set of four patterns shown in Figure 1. The letterlike quality of the patterns was irrelevant to the study: Patterns were chosen to be as identifiable in the absence of a nontarget as possible, on the basis of pilot testing. Thus, the form of each pattern was not considered as important as the fact that the patterns contained as few overlapping pins as possible. Each pattern was presented for $70 \mathrm{msec}$ in a static mode in which all of the pins in the pattern were activated simultaneously (Craig, 1980). As is shown in Figure 1, each pattern occupied an area on the array that was five columns by nine rows, or $9.6 \mathrm{~mm}$ wide by $9.6 \mathrm{~mm}$ high. In addition, the patterns were activated at one of two locations on the array: Rows 1-9 (Location 1, or L1) or Rows 10-18 (Location 2, or L2). On any given trial, the target and the nontarget could occupy identical locations, or the centers of the patterns could be separated by nine 
rows $(10.8 \mathrm{~mm})$. Pins in Rows 19 and 20 remained inactive. The total area covered by both locations was $9.6 \mathrm{~mm}$ wide by $20.4 \mathrm{~mm}$ high.

Procedure. The observers were seated with their left index fingers on the tactile array in front of them. Visual representations of the patterns in Figure 1 were placed above the first two number keys on a standard IBM computer keyboard. Patterns A and B were associated with response " 1 ," and Patterns $C$ and D were associated with response " 2 ." The observers used their right hands to initiate trials at their own pace and to respond by pressing the appropriate key. The observers wore earplugs and earphones emitting static noise to eliminate auditory information from the array. To allow the observers to precisely position their fingers on the array, a positioning stimulus was available at the observer's command between trials. The positioning stimulus consisted of the first and last pins in the top and bottom rows simultaneously activated for about $8 \mathrm{sec}$.

The observers were told that on each trial a pair of patterns would be presented sequentially. The observers were asked to respond to one of the patterns (the target) and to ignore the other pattern (the nontarget). Approximately one fourth of the trials were SC trials (identical target and nontarget), approximately one fourth were RC trials (different target and nontarget having the same response), and the remaining half were RI trials (different target and nontarget having different responses). On some trials, the target preceded the nontarget $(+130-m s e c$ stimulus onset asynchrony, or SOA), and on other trials, the nontarget preceded the target $(-130-\mathrm{msec}$ SOA). A third condition, in which the nontarget was absent, was also tested. The observers were informed of the target's location before each trial, but the nontarget was presented randomly to either L1 or L2. Trial-by-trial feedback was provided, consisting of either the word correct or the word incorrect appearing on the computer monitor in front of the observer. The trials were presented in blocks of 50 and were blocked by target location and by SOA condition (including the target-alone condition) in random order. Data were collected over 12 sessions ( 600 trials per SOA condition for each observer).

\section{Results and Discussion}

The percentage of correct target categorizations (PC) was computed for each observer. PC in the absence of a nontarget was higher at $\mathrm{L} 1$ than at $\mathrm{L} 2$ for 4 observers (12\% higher on average), as had been found previously
(Horner, 1997), but was similar at L1 and L2 for 3 observers. Averaged across observers, $\mathrm{PC}$ in the absence of a nontarget was $88 \%$ at $\mathrm{L} 1$ and $82 \%$ at $\mathrm{L} 2$. Although this $6 \%$ difference in accuracy was not statistically significant, it was in the direction of higher accuracy at Ll. Furthermore, the differences in performance across locations among participants only existed for PC in the absence of a nontarget: As will be discussed below, all the participants showed the same types of location effects when targets were categorized in the presence of a nontarget. Performance in the presence of nontargets was similar for all the observers, and their data were therefore combined.

$\mathrm{PC}$ in the presence of nontargets was analyzed separately for each trial type (SC, RC, and RI) and each SOA. These data were further analyzed separately for trials in which nontargets were at SLoc or at DLoc. The results in each condition were not significantly different when targets were at L1 and L2 $(p>.05)$. Therefore, the data for each location were combined, and PC averaged across observers is shown for each condition in Figure 2. Each $\mathrm{SC}$ and $\mathrm{RC}$ data point represents at least 990 trials, and each RI data point represents at least 2,038 trials. The data for individual observers were similar to those in Figure 2: The standard errors of the mean for each data point ranged from $2 \%$ to $4 \%$ and did not differ systematically across SOA, trial type or nontarget location.

The data in Figure 2 were analyzed, using a $2 \times 2 \times$ 3 (nontarget location $\times \mathrm{SOA} \times$ trial type) repeated measures analysis of variance (ANOVA). Only the main effects and interactions reaching at least the .05 level of significance are discussed. There was a main effect of SOA $[F(1,6)=16.74, p<.01]$, showing lower accuracy when nontargets followed the target (backward interference) than when nontargets preceded the target (forward interference), a result found in previous studies using the 4to-2 paradigm with stationary spatial patterns (Craig,

\section{Static Patterns}

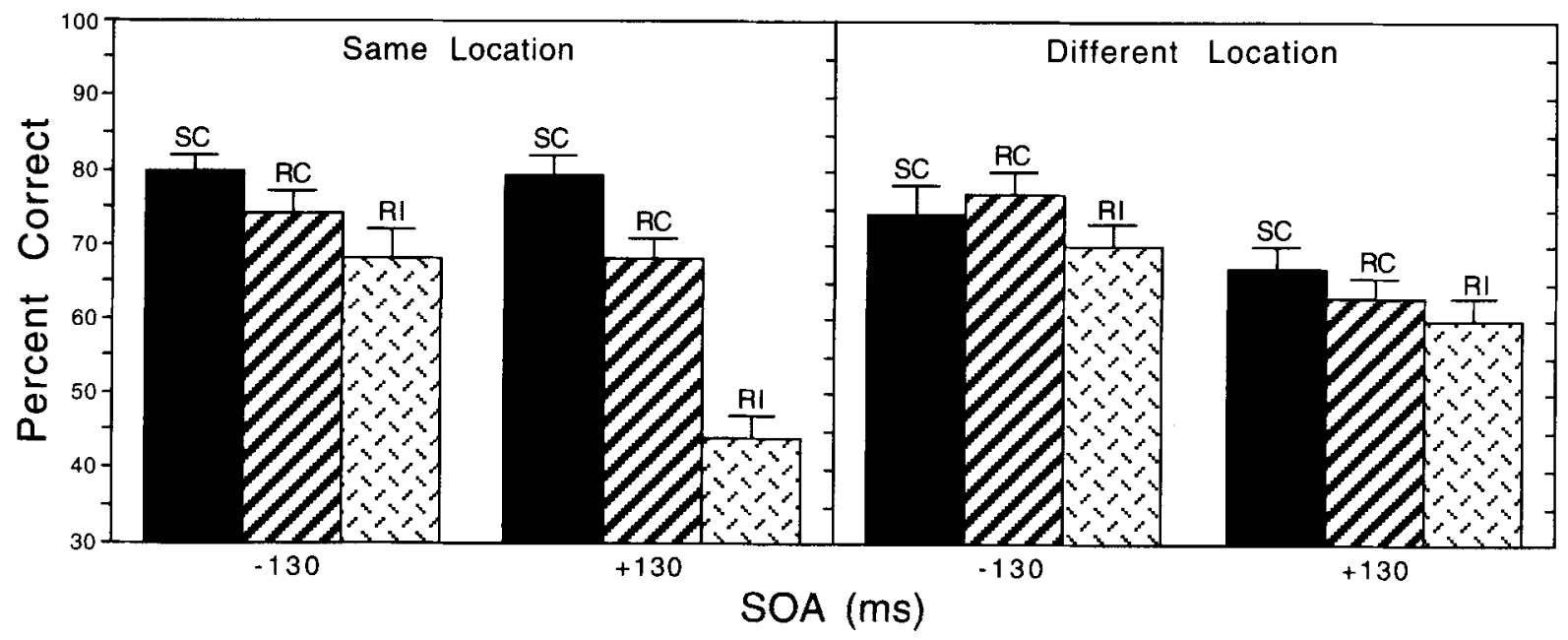

Figure 2. Percentage of correct target categorizations as a function of stimulus onset asynchrony (SOA) in Experiment 1 when targets and nontargets were at the same location (left panel) and when they were at different locations (right panel). Performance at each SOA is shown separately for stimulus compatible (SC), response compatible (RC), and response incompatible (RI) trials. 
1995; Craig \& Evans, 1995). Greater backward than forward interference has been attributed to the nontarget's being more strongly represented than the target at the time the observer responds: Observers may occasionally respond with the more strongly represented trailing nontarget pattern. In addition, greater backward than forward interference has been found in studies of temporal masking, in which backward masking (a temporally trailing masker) leads to lower identification performance than forward masking (a temporally leading masker; Craig, 1976, 1978, 1980, 1982, 1985; Evans, 1987). In these studies, greater backward interference has been attributed to an integration/interruption model of masking. There was also a main effect of trial type $[F(2,12)=65.15$, $p<.01]$ and an interaction between trial type and SOA $[F(2,12)=6.40, p<.02]$, indicating that the difference between trial types was greater when nontargets followed targets than when nontargets preceded targets.

The main question was whether varying the nontarget's location would affect performance on SC, RC, and $\mathrm{RI}$ trials. The interaction between trial type and nontarget location was significant $[F(2,12)=25.79, p<.01]$, reflecting the fact that the difference between trial types was greater when nontargets were at SLoc than when they were at DLoc. In fact, Tukey HSD tests (at a .05 significance level) on the DLoc data showed no significant differences between the three trial types at either SOA. For the SLoc data, there were no significant differences between SC and RC trials at either SOA, a result similar to that of Craig (1995). However, the SLoc data did show a significant difference between RC and RI trials at the +130 -msec SOA, suggesting that response competition caused interference when the nontarget followed the target. The SLoc data showed no significant difference between RC and RI trials at the -130 -msec SOA, although there was a significant difference between SC and RI trials.

The results above indicate that varying the nontarget's location did affect performance, but primarily at the +130 -msec SOA - that is, when the nontarget temporally trailed the target (when backward interference occurred). Tukey HSD tests on the $+130-\mathrm{msec}$ data showed that performance on SC trials declined significantly when nontargets were at DLoc, as compared with SLoc, but performance on RI trials improved significantly when nontargets were at DLoc, as compared with SLoc. Performance on $\mathrm{RC}$ trials did not change significantly as a function of nontarget location. The most likely interpretation of these results is that changing location on the distal pad of the finger affects the perceived shape of patterns, at least to some extent, and that nontargets at a separate location may be perceived differently than nontargets at the SLoc. Indeed, the observers reported that in the absence of nontargets, targets felt somewhat different at L1 and L2. Furthermore, 4 of the observers were more accurate in categorizing targets at $\mathrm{L} 1$ than at $\mathrm{L} 2$ in the absence of nontargets.

Spatial patterns may feel somewhat different at $\mathrm{L} l$ and L2 because of differences in the density of innervation at the two locations (Johansson \& Vallbo, 1979; Vallbo \& Johansson, 1984). A trailing nontarget that is identical to the target but at a different location may feel like a different pattern. How different it may feel is unknown, and it may, in fact, evoke a target response still, a nontarget response, or neither a target nor a nontarget response. Either of the latter two occurrences could interfere to some degree with target categorization on SC trials. A similar situation may hold true on RC trials; nontargets at DLoc may evoke responses different from those evoked at SLoc. However, in the case of RC trials, any changes in the responses evoked by nontargets as a function of location did not result in a significant change in performance. On RI trials, nontargets at DLoc may be less likely to elicit competing responses than would nontargets at SLoc, and observers may be less likely to respond with the nontargets at DLoc.

The foregoing discussion implies that observers have a relatively wide spotlight of attention. If nontargets at DLoc evoke responses that interfere with target categorization, observers are not restricting their attention solely to the target location. According to this view, at least some of the changes in trial type performance that occur when presenting nontargets at DLoc are due to alterations in the kinds of responses evoked by the nontarget. As was mentioned above, nontargets may evoke different responses at DLoc because the density of innervation varies between the two locations. How might one determine whether differences in innervation density at $\mathrm{Ll}$ and $\mathrm{L} 2$ were responsible for the changes in performance for trial types as a function of location? One approach is to determine whether performance continues to vary with location when the perception of the patterns does not depend on their shapes; therefore, the influence of the different innervation densities at $\mathrm{L} 1$ and $\mathrm{L} 2$ should be reduced (Experiment 2). Another approach is to examine whether performance varies with location when skin locations that do not differ appreciably in density of innervation are used (Experiment 3 ).

\section{EXPERIMENT 2}

Experiment 1 showed that varying the location of the nontarget on the distal pad of the finger modified performance. However, responses were based on perceiving the spatial features of patterns, a task that is likely to be strongly affected by the density of innervation of the skin. Changing locations may have less of an effect if responses are based not on perceived shape, but on perceived direction of motion. Specifically, performance on $\mathrm{SC}, \mathrm{RC}$, and RI trials may not depend on whether patterns are presented to SLoc or DLoc on the distal pad of the finger if observers are judging direction of motion. In Experiment 2, the stimuli were bar-shaped patterns that moved in four possible directions across the distal pad of the finger. The observers responded " 1 " if the target moved up or left and "2" if it moved down or right. Comparing the results of Experiments 1 and 2 may shed 
light on the extent to which the Experiment 1 results depended on the processing of spatial features.

Another reason for using moving patterns, rather than stationary patterns, was to raise overall performance. Movement has long been recognized as an effective stimulus for the skin (Katz, 1989), and moving patterns should be easier to perceive than stationary spatial patterns. If so, performance at L2 may more closely approximate performance at $\mathrm{Ll}$, perhaps further reducing the influence of differences in innervation density between $\mathrm{LI}$ and L2. If the effects of location found in Experiment 1 disappear under these conditions, it would suggest that innervation density may account for the results of Experiment 1.

A final reason for using moving patterns is that they more closely approximate the usual way in which the skin processes information. For example, patterns move across the skin during tactual exploration of an object's surface and when tactile communication systems, such as Braille and the Optacon, are used. Moving patterns have been used in a number of previous studies to examine both masking and response competition (Craig \& Evans, 1995; Evans \& Craig, 1991, 1992; Evans et al., 1992; Rinker \& Craig, 1994). However, in these studies patterns were presented either to locations on separate fingers or to the SLoc.

\section{Method}

Participants. Seven female students served as trained observers; all had received practice in tasks similar to the present one. Four of the 7 observers had also participated in Experiment 1. Participants either were paid or received course credit for participation.

Apparatus. The same apparatus was used as that in Experiment 1.

Stimuli. The targets and nontargets were randomly selected with replacement from a set of four barlike moving stimuli like those depicted in Figure 3. The arrows indicate the direction and distance of movement. Vertical bars were nine rows $(9.44 \mathrm{~mm})$ in length, and horizontal bars were five columns $(9.16 \mathrm{~mm})$ in length. Movement of the bar stimuli (left, right, up, or down, ) at either L1 or L2 was simulated on the fingerpad by activating pins in a column-by-column sequence or a row-by-row sequence. The total duration of each stimulus, from the onset of the first row or column to the offset of the last row or column, was $118 \mathrm{msec}$.

Procedure. The same procedure was used as that in Experiment 1 , except that the observers were told to respond to the target on the basis of its perceived movement, rather than its shape, and the number of trials per block was increased to 70 . The increase in pattern duration from $70 \mathrm{msec}$ in Experiment 1 to $118 \mathrm{msec}$ in Experiment 2 led to an increase in the SOAs used from $\pm 130 \mathrm{msec}$ in Experiment 1 to $\pm 156 \mathrm{msec}$ in Experiment 2. No practice sessions were included, because the observers already had experience in categorizing static patterns on the array and the resulting data showed no learning effects. Data were collected over seven sessions (490 trials per SOA condition for each observer).

\section{Results and Discussion}

Unlike the results of Experiment 1, PC in the absence of a nontarget was not significantly different at $\mathrm{Ll}$ and $\mathrm{L} 2$ for all the observers $(95 \%$ at $\mathrm{L} 1$ and $93 \%$ at $\mathrm{L} 2$, averaged across observers). This similarity in performance was probably due to the relative ease of perceiving direction of motion, as compared with identifying the spatial features of patterns, and suggests that the perception of movement was similar at the two locations. As in Experiment $1, \mathrm{PC}$ in the presence of nontargets was analyzed by trial type, SOA, and nontarget location (SLoc or DLoc). $P C$ averaged across observers is shown for each condition in Figure 4. Each SC and RC data point represents at least 385 trials, and each RI data point represents at least 840 trials. The standard errors of the mean for each data point ranged from $3 \%$ to $8 \%$ and did not differ systematically across SOA, trial type, or nontarget location.

Performance for all trial types was generally better for moving bars than for static patterns. A $2 \times 2 \times 3$ (nontarget location $\times$ SOA $\times$ trial type) ANOVA revealed
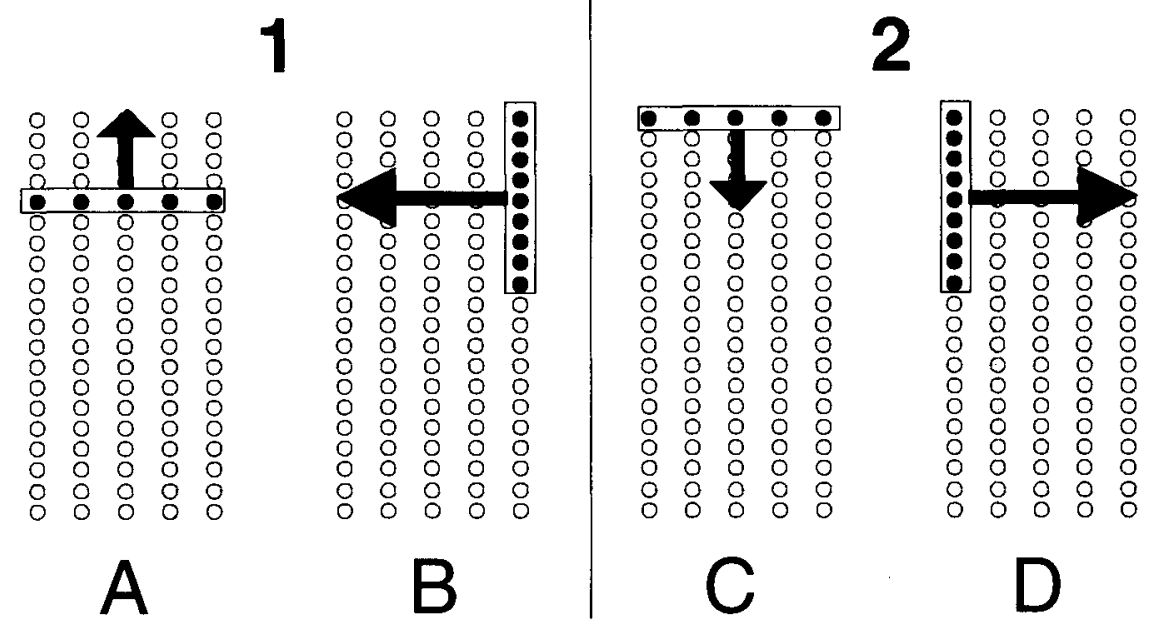

Figure 3. Representations of the moving bar patterns used as targets and nontargets in Experiment 2 as they would appear at the top of the distal pad of the finger (L1). Filled circles indicate which pins were activated, and the arrows indicate the direction and extent of movement. 


\section{Moving Patterns}

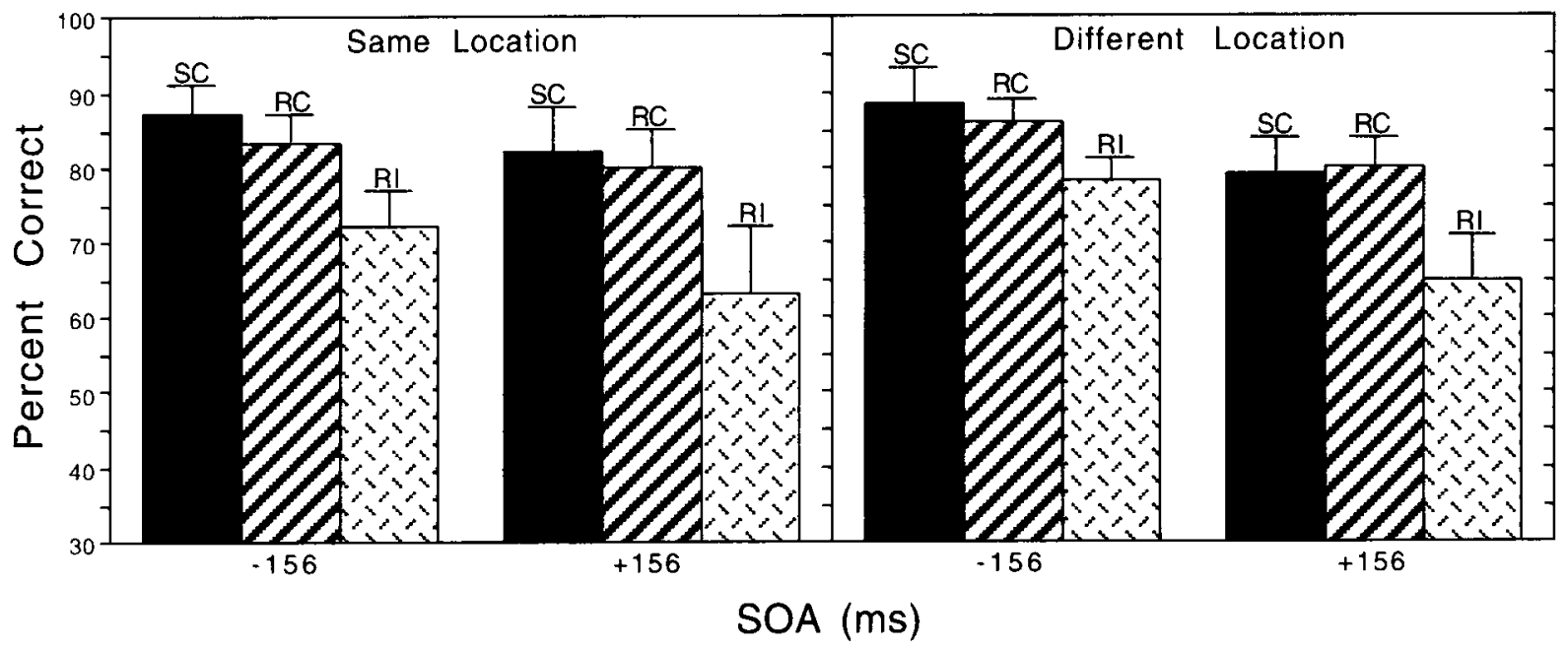

Figure 4. Percentage of correct target categorizations as a function of stimulus onset asynchrony (SOA) in Experiment 2 when targets and nontargets were at the same location (left panel) and when they were at different locations (right panel). Performance at each SOA is shown separately for stimulus compatible (SC), response compatible (RC), and response incompatible (RI) trials.

only main effects of trial type $[F(2,12)=6.13, p<.01]$ and SOA $[F(1,6)=6.62, p<.04]$. As in Experiment 1 , there was greater backward than forward interference. However, unlike Experiment 1, the effects of trial types were similar at both SOAs, as was reflected by the lack of an interaction between trial type and SOA $[F(2,12)=$ $0.67, p>.05]$. The similarity in the effects of trial type at the two SOAs may be due to using both moving patterns and longer SOAs in Experiment 2. With moving patterns, responses are based on the perceived direction of motion, rather than on the shapes of the patterns. Apparent motion between the target and the nontarget might interfere with target categorization, and the strength of the apparent motion generated may be independent of the temporal order of the target and the nontarget, thus producing equivalent forward and backward interference (Craig \& Evans, 1995).

As in Experiment 1, the main question was whether varying the nontarget's location would affect the relative performance levels on SC, RC, and RI trials. Unlike Experiment 1 , there was no significant interaction between trial type and nontarget location $[F(2,12)=2.07, p>.05]$. Thus, performance levels on SC, RC, and RI trials were not significantly affected by the nontarget location, contrary to the result for static patterns. Tukey HSD tests (at the .05 significance level) conducted by nontarget location and by SOA showed no significant differences in performance between SC and RC trials in any condition. However, in all the conditions, there were significant differences between RC and RI trials, indicating the important role that response competition played in interfering with performance, as in Experiment 1. Furthermore, the fact that response competition occurred when nontargets were at DLoc suggests that the observers were un- able to restrict their attention to the target location. A relatively wide spotlight of attention for perceiving moving patterns is also consistent with the results of Experiment 1 .

The results of Experiment 1 were believed to be due to a difference in the density of innervation between $\mathrm{Ll}$ and L2. This difference may have caused shapes at the two locations to be perceived differently. The logic behind Experiment 2 was to use the same two locations but to base responses on direction of motion, rather than on shape. The fact that basing responses on direction of motion eliminated the effect of location supports the idea that the results of Experiment 1 were due to innervation density differences. An alternative approach was used in Experiment 3: Responses were based on shape, but the locations did not vary appreciably in density of innervation.

\section{EXPERIMENT 3}

Experiment 3 used the same general procedure as that in Experiment 1, but patterns were presented to two locations on the medial pad of the index finger, rather than to two locations on the distal pad. The skin of the medial pad does not vary substantially in density of innervation (Johansson \& Vallbo, 1979; Vallbo \& Johansson, 1984). If changing the location alters the perceived shapes of patterns because of differences in density of innervation at the two locations, one would not expect location to affect performance on the medial pad.

The results of both Experiments 1 and 2 indicated that response competition played a much larger role than did masking in limiting performance. Presumably, the nontarget response was available on each trial, and the observers occasionally responded with the nontarget, rather than with the target. This would harm performance only 
on RI trials, since only on these trials was the nontarget response different from the target response. One limitation of the 4-to-2 paradigm is that with only two responses available, it was impossible to determine whether the observers had access to the nontarget response and responded incorrectly or whether they had no information about the nontarget's identity and were simply guessing. To avoid this limitation, Craig (1995) implemented a 6to-3 paradigm, in which there were six patterns and three available responses. By analyzing incorrect responses on RI trials, he determined that observers selected the nontarget response more often than the remaining response. Experiment 3 also used a 6-to-3 paradigm to determine whether observers select the nontarget or just guess on incorrect trials. Furthermore, incorrect responses were analyzed as a function of location, to determine whether information about the nontarget's identity changes with its location. Another advantage of using the 6-to-3 paradigm is to ensure that the results of Experiments 1 and 2 were not due to limiting observers to only two responses.

\section{Method}

Participants. Five female students served as trained observers, 2 of whom had also participated in Experiment 2. All of the observers were paid for their participation.

Apparatus. The same apparatus was used as that in Experiments 1 and 2 .

Stimuli. The targets and nontargets were randomly selected with replacement from the set of six patterns shown in Figure 5. As in Experiment 1, the patterns were pilot tested to be as identifiable in the absence of a nontarget as possible, with as few overlapping pins as possible. Each pattern was presented for $50 \mathrm{msec}$ in the static mode. The patterns were activated at one of two locations: Rows 1-7 (Location 1, or L1) or Rows 8-14 (Location 2, or L2). On any given trial, the target and the nontarget could occupy identica! locations, or the centers of the patterns could be separated by 7 rows $(8.4 \mathrm{~mm})$. Pins in Rows $15-20$ remained inactive. The total area covered by both locations was $9.6 \mathrm{~mm}$ wide by $15.6 \mathrm{~mm}$ high.

Procedure. The same procedure was used as that in Experiment 1 , except that the observers placed the medial pad of their index finger on the array, rather than their distal pad. Patterns $A$ and $B$ were associated with Response 1 , Patterns C and D with Response 2, and Patterns $E$ and $F$ with Response 3. Only a single SOA was tested $(+88 \mathrm{msec})$, along with a condition in which the nontarget was absent. Data were collected over 10 sessions ( 750 trials per condition for each observer).

\section{Results and Discussion}

$\mathrm{PC}$ in the absence of nontargets was not significantly different at $\mathrm{LI}$ and $\mathrm{L} 2$ for all observers $(86 \%$ at $\mathrm{L} 1$ and $85 \%$ at $L 2$, averaged across observers). This similarity in performance was probably due to a similarity in density of innervation at the two locations and suggests that the perception of patterns was similar at the two locations. $\mathrm{PC}$ in the presence of nontargets was analyzed by trial type and nontarget location. The results in each condition were similar when targets were at $\mathrm{L} 1$ and $\mathrm{L} 2$. Therefore, the data for each location were combined, and PC averaged across observers is shown for each condition in Figure 6. Each $\mathrm{SC}$ and RC data point represents at least 575 trials, and each RI data point represents at least 2,480 trials. The data for individual observers were similar to those in Figure 2: The standard errors of the mean for each data point ranged from $3 \%$ to $5 \%$ and did not differ systematically across trial type or nontarget location.

The relative performance levels between $\mathrm{SC}, \mathrm{RC}$, and RI trials were similar to the results of Experiment 2. A 2 $\times 3$ (nontarget location $\times$ trial type) ANOVA revealed only a main effect of trial type $[F(2,8)=53.37, p<.001]$. The lack of a significant main effect of nontarget location and the lack of an interaction between nontarget location and trial type reflect the similarity in SLoc and DLoc results seen in Figure 6. Furthermore, Tukey HSD tests indicated no significant differences between SLoc and DLoc for any of the trial types $(p>.05)$. Thus, when static patterns were presented to locations that were similar in density of innervation, nontarget location had no effect on performance. This result presumably occurred

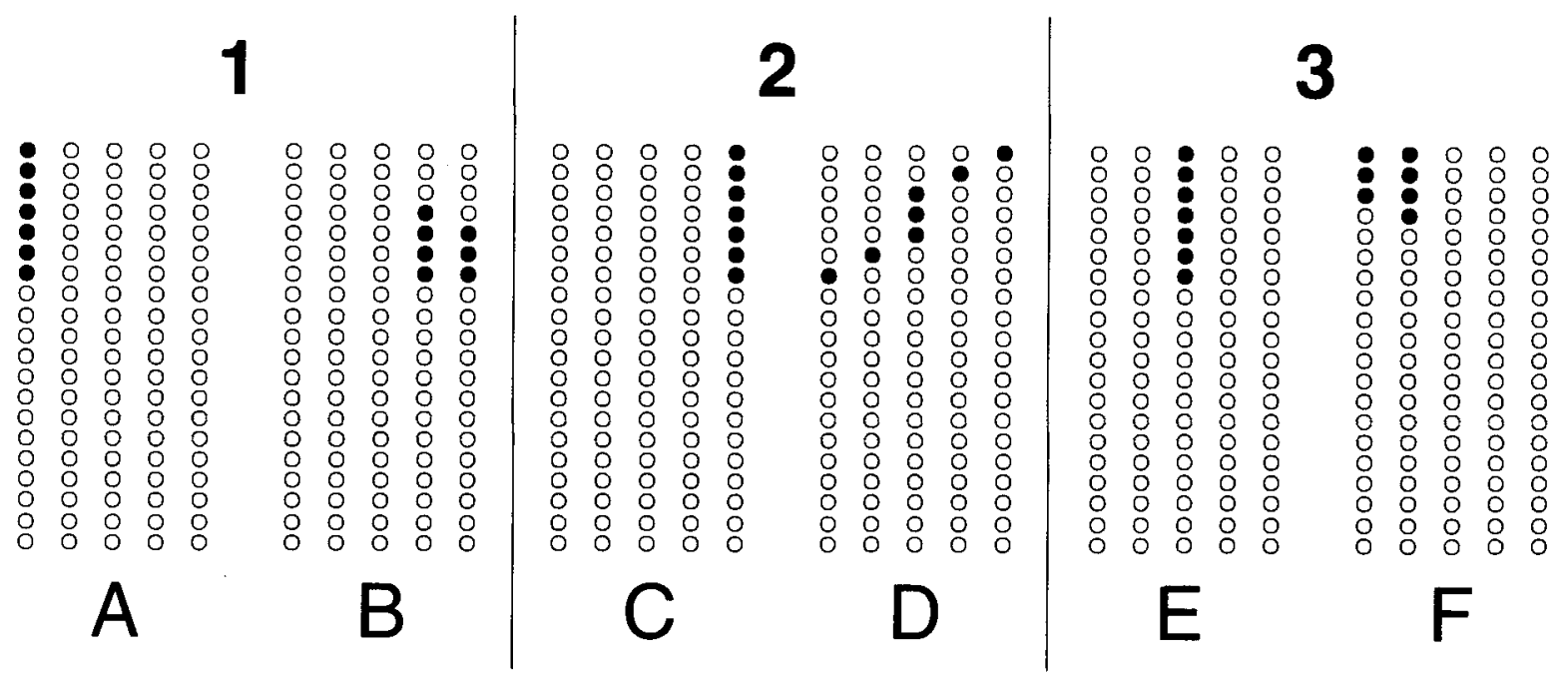

Figure 5. Representations of the patterns used as targets and nontargets in Experiment 3 as they would appear at the top of the medial pad of the finger (L1). Filled cireles indicate active pins, and unfilled circles indicate inactive pins. 


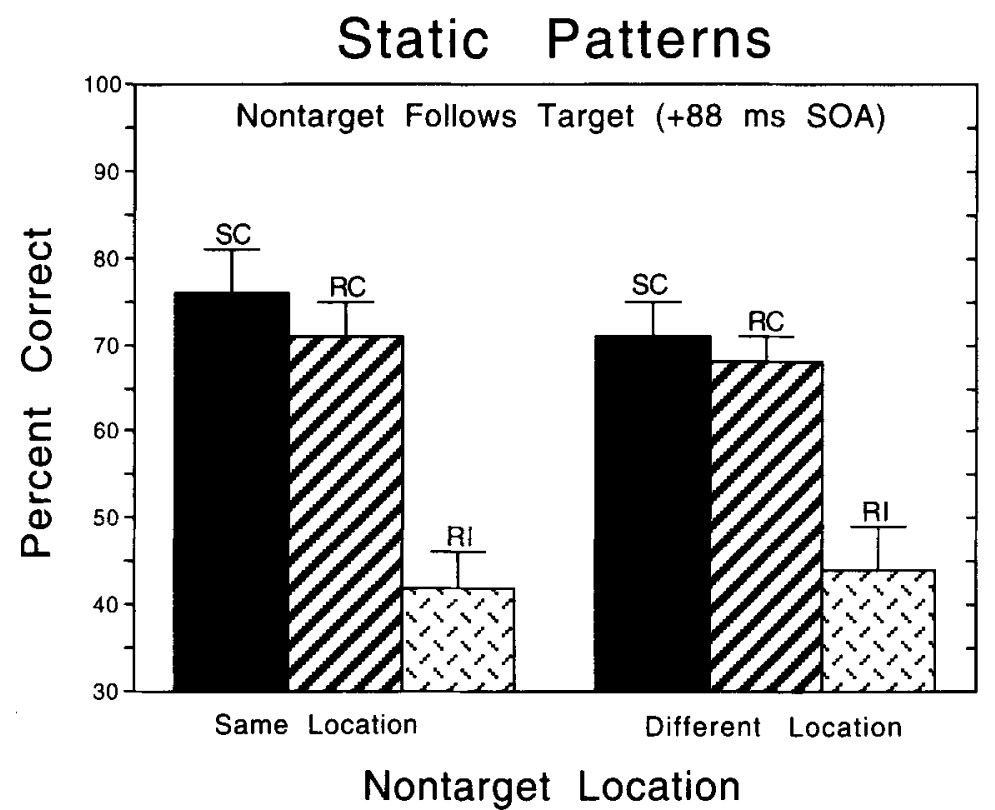

Figure 6. Percentage of correct target categorizations in Experiment 3 when targets and nontargets were at the same location and when they were at different locations. Performance is shown separately for stimulus compatible (SC), response compatible (RC), and response incompatible (RI) trials.

because the perceived shapes of static patterns in Experiment 3 remained the same regardless of their location.

The difference in results between Experiments 1 and 3 may appear surprising, given the fact that in Experiment 1 , there was no significant difference across locations for PC in the absence of a nontarget (only a $6 \%$ difference when averaged across observers). However, this $6 \%$ difference tends to obscure the fact that one group of observers showed large differences across locations for PC in the absence of a nontarget, whereas one group did not. Furthermore, the performance of the two groups of observers in Experiment 1 differed only for $\mathrm{PC}$ in the $\mathrm{ab}-$ sence of a nontarget - that is, only when a single pattern was presented to one of the two locations. Both groups of observers performed similarly when categorizing target patterns in the close temporal proximity of nontarget patterns, and each observer's performance showed that varying trial type had different effects on PC when nontargets were at SLoc versus DLoc. It may be that different densities of innervation at separate locations play a more important role in perception when two patterns are presented in close temporal proximity than when a single pattern is presented. When a target is categorized in the absence of nontargets, observers may be better able to cope with differences in perceived shape that are due to innervation density differences at the separate locations.

There was also no significant difference between SC and RC performance at either SLoc or at DLoc $(p>.05)$. However, the differences in performance between RC and RI trials were significant at both SLoc and DLoc, indicating, as in Experiments 1 and 2, that response compe- tition played a significant role in limiting performance. The primary reason for using a 6-to-3 paradigm was to determine whether observers responded with the nontarget (or simply guessed) on incorrect trials and whether such responding varied with the nontarget's location. If observers responded with the nontarget, the percentage of incorrect RI trials on which observers selected the nontarget response would exceed those on which they selected the remaining response. Averaged across all observers, the percentage of nontarget responses on incorrect RI trials was $74 \%$ for SLoc and $67 \%$ for DLoc. Clearly, on incorrect RI trials, the observers responded more often with the nontarget category than with the remaining category. Furthermore, there was only a $7 \%$ change in such responding when nontarget location varied, reflecting the similarity in the location results in Figure 6. As in Experiments 1 and 2, the results are consistent with the view that the observers were unable to restrict their attention to the target location.

\section{GENERAL DISCUSSION}

The main results of the present study can be summarized as follows: (1) Varying location on the distal pad of the index finger affects the perception of stationary patterns, but not of moving patterns; (2) although the perception of stationary patterns is affected by varying location on the distal pad of the index finger, there is no such effect on the medial pad; (3) response competition plays a major role in limiting the perception of both stationary and moving patterns, at least for the sets of pat- 
terns tested in the present study; and (4) regardless of the type of vibrotactile pattern, observers are unable to restrict their attention completely to a single location.

Differences in innervation density on the distal pad of the finger may play a more important role when perception of patterns depends on processing spatial features than when perception depends on processing movement. Processing of spatial features may be more disrupted by changes in innervation density than is judging the direction of motion of patterns. Loomis (1981 a, 1981b) proposes that the skin behaves as a low-pass spatial filter. According to this view of the skin, changing innervation density may slightly modify the spatial filtering properties of the skin, and such a modification may have a more pronounced effect on the processing of spatial features than on determining the direction of motion. Presumably, no such modification of spatial filtering properties occurs between adjacent locations on the medial pad of the finger because these locations have similar densities of innervation (Johansson \& Vallbo, 1979; Vallbo \& Johansson, 1984). According to this view, patterns are unaffected by varying locations on the medial pad because these locations have similar spatial filtering properties. Under such conditions, information about shape may be somewhat independent of location. However, on the distal pad where adjacent locations may have slightly different filtering properties, just the opposite may be true; shape information may depend on location to some degree.

Previous effects of testing adjacent locations on the distal pad of the finger, using Optacon patterns (Hill, 1974; Horner, 1995, 1997), were almost certainly influenced by the differences in density of innervation at the top and the bottom of the distal pad. Hill presented letters sideways on the Optacon display moving up or down the distal pad of the index finger and measured observers' reading rates. He found that reading rates were slower with the bottom half than with the top half of the display and concluded that the top of the pad was responsible for reading skill. Horner (1995) found that discrimination performance declined with increasing distance between spatial patterns on the distal pad of the index finger. There was also a bias toward responding different when sameshape patterns were presented to separate locations, despite the fact that observers received feedback and knew only half the trials were different. Both of the above studies used the Optacon display and, hence, used vibratory patterns whose spatial details are probably being encoded primarily by FAI (RA) afferent units (Gardner \& Palmer, 1989a, 1989b, 1990). These units show a more pronounced reduction in density from the top to the bottom of the distal pad of the finger than do FAII (PC) units, which have generally larger receptive fields (Johansson \& Vallbo, 1979; Vallbo \& Johansson, 1984). The perception of nonvibratory spatial patterns may be influenced also by differences in density of innervation across the distal pad of the finger. Nolan and Kederis (1969) found that the locations of dots in Braille characters affected recognition. Lower cell characters were recognized more slowly than upper cell characters, and dots were missed more frequently when at the bottom than when at the top of the cell. Braille characters are probably best encoded by SAI afferent units (Johnson \& Lamb, 1981), which also show a pronounced reduction in density from the top to the bottom of the distal pad of the finger (Johansson \& Vallbo, 1979; Vallbo \& Johansson, 1984).

Regardless of the filtering properties of adjacent locations on a single fingerpad, a pattern at a neighboring location can still interfere with target processing. Such interference seems to be due primarily to response competition, in which representations of both the target and the nontarget are available and the observer mistakenly responds with the nontarget. Thus, it appears to be difficult to narrow the attentional spotlight solely to the target location. The amount of interference caused by a nontarget at DLoc is particularly surprising, given the fact that observers have the additional cue of location to help determine which pattern is the target. In Horner's (1997) study of temporal masking on the distal pad of the finger, it appeared that maskers at adjacent locations interfered with target identification at two stages in processing, an early stage (lateral masking) and a later stage (response competition). When nontargets were at DLoc in the present study, response competition was clearly operating, but lateral masking cannot be entirely ruled out. On trials in which the target and the nontarget differed in shape, responding with the nontarget would have reduced performance on RI trials, but it would have increased performance on RC trials, since responding with the nontarget would have been a correct response. Thus, comparing performance on $\mathrm{RC}$ trials with performance on SC trials may underestimate the amount of interference that is due to masking (Craig, 2000).

The fact that varying the location of a spatial pattern affected its perception when presented to the distal pad of the finger, but not to the medial pad, probably reflects differences in the density of innervation for these two areas of skin. This result may also reflect how these areas of skin are used differently in exploring the surface features of an object. In exploring an object tactually, the palm and fingers may be used to scan the various surfaces of the object. However, when a feature of interest is located on the surface of an object, the tip of the distal pad may be used to further investigate details of the feature. For example, when inspecting a coffee mug for a crack, the fingers and palm may be used, but when the crack is located, the tip of the finger may be employed for further inspection.

\section{REFERENCES}

Bliss, J. C., Katcher, M. H., Rogers, C. H. \& Shepari, R. P. (1970) Optical-to-tactile image conversion for the blind. IEEE Transactions on Man-Machine Sustems, MMS-11, 58-64.

Craig. J. C. (1976). Vibrotactile letter recognition: The effects of a masking stimulus. Perception \& Psichophwics, 20. 317-326. 
Craig, J. C. (1978). Vibrotactile pattern recognition and masking. In $\mathrm{G}$. Gordon (Ed.), Active touch-the mechanism of recognition of ohjects by manipulation: A multi-disciplinary approach (pp. 229-242). Oxford: Pergamon.

Cralg, J. C. (1980). Modes of vibrotactile pattern perception. Journal of Experimental Psychology: Human Perception \& Performance, 6. $151-166$.

CRAIG, J. C. (1982). Vibrotactile masking: A comparison of energy and pattern maskers. Perception \& Psychophysics, 31. 523-529.

CraIG. J.C. (1983). The role of onset in the perception of sequentially presented vibrotactile patterns. Perception \& Psvchophysics, 34, 421-432.

CRAIG. J. C. (1985). Tactile pattern perception and its perturbations. Journal of the Acoustical Society of America, 77, 238-246.

Cralg, J. C. (1989). Interference in localizing tactile stimuli. Perception \& Psvchophysics, 45, 343-355.

CralG, J. C. (1995). Vibrotactile masking: The role of response competition. Perception \& Psvchophysics, 57, 1190-1200.

CRAIG, J. C. (2000). Processing of sequential tactile patterns: Effects of a neutral stimulus. Perception \& Psichophisics, 62, 596-606.

Craig, J. C. \& Evans. P. M. (1987). Vibrotactile masking and the persistence of tactual features. Perception \& Psichophvsics, 42. 309-317.

Cralg. J. C.. \& Evans, P. M. (1995). Tactile selective attention and temporal masking. Perception \& Psichophisics, 57, 511-518.

Driver, J.. \& Bayl.Is. G. C. (1991). Target-distractor separation and feature integration in visual attention to letters. Acta Psychologica. 76, $101-119$

ERIKSin, C. W., \& Hoffman, J. E. (1973). The extent of processing of noise elements during selective encoding from visual displays. Perception \& Pswchophisics, 14, 155-160.

Evans. P. M. (1987). Vibrotactile masking: Temporal integration, persistence, and strengths of representations. Perception \& Psychophisics, 42, 515-525.

Evans, P. M.. \& Cralci, J. C. (1986). Temporal integration and vibrotactile backward masking. Journal of Experimental Psichology: Human Perception \& Performance. 12, 160-168.

Evans. P. M., \& CRAlki, J. C. (1991). Tactile attention and the perception of moving tactile stimuli. Perception \& Psuchophrsics, 49, 355-364.

Evans. P. M., \& CrAKk. J. C. (1992). Response competition: A major source of interference in a tactile identification task. Perceprion \& Psichophisics. 51, 199-206.

Evans, P. M., Cral(i. J. C.. \& Rinker. M. A. (1992). Perceptual processing of adjacent and nonadjacent tactile nontargets. Perception \& Psichophrsics, 52, 571-581.

Gardner, E. P.. \& Palmer. C. I. (1989a). Simulation of motion on the skin: I. Receptive fields and temporal frequency coding by cutaneous mechanoreceptors of Optacon pulses delivered to the hand. Journal of Neurophrsiology, 62, 1410-1436.
Gardner, E. P., \& Palmer, C. I. (1989b). Simulation of motion on the skin: II. Cutaneous mechanoreceptor coding of the width and texture of bar patterns displaced across the Optacon. Journal of Neurophysiology, 62, 1437-1460

Gardner, E. P., \& Palmer, C. I. (1990). Simulation of motion on the skin: III. Mechanisms used by rapidly adapting (RA) cutaneous mechanoreceptors in the primate hand for spatiotemporal resolution and two-point discrimination. Journal of Neurophysiologv, 63, 841-859.

HIt.L., J. W. (1974). Limited field of view in reading lettershapes with the fingers. In F. A. Geldard (Ed.), Cutaneous communication systems and devices (pp. 95-105). Austin, TX: Psychonomic Society.

HORNER, D. T. (1995). The effect of location on the discrimination of spatial vibrotactile patterns. Perception \& Psychophysics, 57, 463-474.

HORNER, D. T. (1997). The effect of shape and location on temporal masking of spatial vibrotactile patterns. Perception \& Psychophysics, 59. $1255 \cdot 1265$.

Johansson, R. S., \& Vallbo, A. B. (1979). Tactile sensibility in the human hand: Relative and absolute densities of the four types of mechanoreceptive units in glabrous skin. Journal of Physiologv, 286, 283-300

JoHnson, K. O., \& LAmB, G. D. (1981). Neural mechanisms of spatial tactile discrimination: Neural patterns evoked by Braille-like dot patterns in the monkey. Journal of Physiology, 310, 117-144.

KATZ, D. (1989). The world of touch (L. E. Krueger, Trans.). Hillsdale, NJ: Erlbaum

LoOMIs, J. M. (1981a). On the tangibility of letters and braille. Perception \& Psvchophvsics, 29, 37-46.

LoOMıs, J. M. ( 198 I b). Tactile pattern perception. Perception, 10, 5-27.

Loomis. J. M., \& APKARIAN-STIELAU. P. (1976). A lateral masking effect in tactile and blurred visual letter recognitjon. Perception \& Psichophisics, 20, 221-226.

Nolan. C. Y.. \& Kederis. C. J. (1969). Perceptual factors in braille word recognition. New York: American Foundation for the Blind

Rinker. M. A., \& CralG, J. C. (1994). The effect of spatial orientation on the perception of moving tactile stimuli. Perception \& Psychophysics, 56, 356-362.

VAli.bo, A. B., \& Johansson, R. S. (1984). Properties of cutaneous mechanoreceptors in the human hand related to touch sensation. Human Neumohiologv, 3, 3-14

WEISFNBERGER, J. M. (1981). Tactile pattern similarity. Unpublished doctoral dissertation, Indiana University.

Wfisfnberger, J. M. \& Craig. J. C. (1982). A tactile metacontrast effect. Perception \& Psychophysics, 31, 530-536.

(Manuscript received October 13, 1999; revision accepted for publication February 20,2000.) 